\title{
\&UIISIBRAGEC
}

\section{LEVANTAMENTO DE SOLUÇÕES CONSTRUTIVAS EM MADEIRA INDUSTRIALIZADA ${ }^{1}$}

\section{LOPES, Shara Carvalho (1); CARMO, Murilo Elias Rosa do (2); SERRA, Sheyla Mara Baptista (3)}

(1) Universidade Federal de São Carlos, shara_cl@hotmail.com (2) Universidade Federal do Rio Grande do Sul, murilo.elias@ mail.uft.edu.br, (3) Universidade Federal de São Carlos, sheylabs@ufscar.br

\begin{abstract}
RESUMO
Devido à competitividade das empresas e a implantação de novas técnicas de gestão da indústria 4.0, a indústria da construção busca a implantação de novas soluções tecnológicas e procedimentos mais sustentáveis, social, ambiental e economicamente. Assim, as soluções tecnológicas em madeira ganham cada vez mais espaço e atraem um olhar otimista para o futuro. $O$ alto índice de qualidade dos elementos de madeira pré-fabricados gera uma descentralização dos canteiros de obra, concentrando neles apenas a montagem das estruturas, e colaborando para a disseminação da construção off-site. Obtém-se uma redução de perdas, de mão-de-obra, de tempo de execução e, consequentemente, diminuição dos custos totais de obra. Logo, para sistematização das soluções existentes no mercado brasileiro, este trabalho teve por objetivo diagnosticar o atual cenário das construções em madeira no Brasil, a fim de identificar as soluções tecnológicas atuais. A metodologia empregada foi a de análise documental, por meio de pesquisa bibliográfica e, em seguida, foram identificadas as empresas que trabalham com os sistemas construtivos estudados. Pode-se concluir que, a madeira começa a recuperar a notoriedade de outros tempos, apoiada nas crescentes preocupações ambientais, nos avanços tecnológicos e nos estudos de suas características.
\end{abstract}

Palavras chave: Soluções tecnológicas em madeira, Sistemas construtivos industrializados, Construção off-site, Construção civil.

\begin{abstract}
Due to the competitiveness of companies and the implementation of new management techniques for industry 4.0, the construction industry seeks to implement new construction solutions and more sustainable procedures, socially, environmentally and economically. Thus, technological solutions in wood gain more space and attract an optimistic look to the future. The high-quality index of the prefabricated wooden elements generates a decentralization of the construction sites, concentrating on them only the assembly of the structures, and contributing to the dissemination of off-site construction. A reduction in losses, labor, execution time and, consequently, a reduction in total labor costs is achieved. Therefore, in order to systematize the existing solutions in the Brazilian market, this work aimed to diagnose the current scenario of wood constructions in Brazil, in order to identify the current technological solutions. The methodology used was that of documentary analysis, through bibliographic research and, then, the companies that work with the studied construction systems were identified. It can be concluded that, the wood begins to recover the notoriety of other times, supported by the growing environmental concerns, in the technological advances and in the studies of its characteristics.
\end{abstract}

Keywords: Technological solutions in wood, Industrialized construction systems, Off-site construction, Construction Industry.

\footnotetext{
${ }^{1}$ LOPES, S. C.; CARMO, M. E. R. do; SERRA, S. M. B. Levantamento de soluções tecnológicas em madeira industrializada no Brasil. In: SIMPÓSIO BRASILEIRO DE GESTÃO E ECONOMIA DA CONSTRUÇÃO, 12., 2021, Maceió. Anais[...] Porto Alegre: ANTAC, 2021. p.1-8. Disponível em: https://eventos.antac.org.br/index.php/sibragec/article/view/51 1. Acesso em: 2 out. 2021.
} 


\section{INTRODUÇÃOO}

A industrialização de um sistema construtivo é classificada como o ápice da racionalização na construção, pois proporciona uma produção em caráter industrial, visando a montagem no canteiro de obras, independente do material em questão (ABDI, 2015). Com isso também se viabiliza um controle mais rigoroso e produtivo com a possibilidade da incorporação de novas tecnologias construtivas.

No contexto das soluções tecnológicas para sistemas construtivos industrializados de madeira estão inseridos as log homes, wood frame e as madeiras engenheiradas, como: madeira laminada cruzada (Cross Laminated Timber - CLT), madeira microlaminada (Laminated Venner Lumber - LVL), madeira laminada colada sob alta pressão e temperatura (Parallel Strand Lumber - PSL), madeira laminada colada (MLC), madeira laminada serrada (Laminated Strand Lumber - LSL), madeira serrada orientada (Oriented Strand Lumber - OSL), madeira laminada pregada (Nail-Laminated Timber - NLT), madeira laminada cavilhada (Dowel Laminated Timber - DLT) e painel de tiras de madeira orientadas (Oriented Strand Board - OSB), que superam os inconvenientes que as madeiras maciças oferecem, como a anisotropia, a fraca durabilidade biológica, a baixa estabilidade global e a baixa resistência ao fogo (MORADPOUR et al., 2018). Esses sistemas construtivos em madeira têm sido amplamente utilizados como material de construção estrutural, seja como substituto ou em conjunto com o concreto e o aço (KUZMAN et al., 2018).

É relevante como nas últimas décadas a preocupação com o uso do solo, a consciência ambiental, a diminuição do uso de recursos florestais e o crescente interesse social em uma construção de baixo consumo de energia alteraram a perspectiva da indústria da madeira em todo o mundo, gerando uma grande oportunidade para seu uso como material de construção (MORADPOUR et al., 2018; RESCALVO et al., 2020). O Brasil vem incorporando gradativamente o processo de produção industrializado de um edifício em madeira, que vai muito além da concepção do projeto, envolvendo elementos variáveis da cadeia de produção: econômicos, logísticos, organizacionais e culturais (ABDI, 2015).

Então, esse trabalho se propõe em identificar as principais soluções tecnológicas em madeira industrializada disponíveis no mercado brasileiro, por meio de pesquisa bibliográfica e documental, garantindo um estudo técnico sobre um assunto ainda pouco difundido no âmbito do mercado nacional. Esta pesquisa possui o intuito de gerar insights aos estudantes, pesquisadores e profissionais da área de construção civil na escolha de qual sistema construtivo e solução tecnológica industrializada em madeira utilizar em sua obra. Adicionalmente, são identificadas e elencadas as principais empresas que atuam tanto na fabricação dos materiais, quanto na concepção e execução das edificações.

\section{MÉTODO DE PESQUISA}

Foi desenvolvida uma pesquisa documental do tipo descritiva e qualitativa que pode ser utilizada em complemento à pesquisa bibliográfica. Para Tumelero (2019), a pesquisa documental visa identificar dados e informações que ainda não foram tratados científica ou analiticamente. Para esses autores, os documentos a serem analisados podem ser delimitados de acordo com um período temporal, e podem ser utilizados para contextualizar um determinado momento da história.

Dessa forma, este trabalho reúne um levantamento das soluções tecnológicas industrializadas em madeira utilizadas atualmente no Brasil e procura identificar as empresas nacionais que fabricam, criam projetos e executam obras deste segmento. $\mathrm{O}$ período de coleta se restringiu a março e abril de 2021. Foram utilizadas pesquisas bibliográficas e documentais com base em manuais, artigos científicos e informações fornecidas nos websites das empresas listadas. As ferramentas de busca utilizadas foram 
Google e Google Scholar para a obtenção do material de referência bibliográfica e informações das empresas. Os termos de busca aplicados no primeiro estágio da pesquisa para identificação dos sistemas construtivos em madeira foram "sistemas construtivos em madeira" e "construção em madeira industrializada". Após a pesquisa inicial, no segundo estágio, foi realizada uma busca para identificar quais destes sistemas são fabricados e executados no Brasil com os termos de busca "sistemas construtivos em madeira no Brasil" e "madeira engenheirada no Brasil".

\section{SISTEMAS CONSTRUTIVOS INDUSTRIALIZADOS EM MADEIRA NO BRASIL}

A partir do cenário delineado, analisou-se as tecnologias industrializadas em madeira empregadas no Brasil, definidas em: sistema wood frame, log home, madeira laminada cruzada e madeiras engenheiradas utilizadas no sistema pilar-viga.

\subsection{Wood frame}

O wood frame é constituído de um sistema de vedação autoportante e leve, cujas paredes são compostas por quadro de elementos de madeira (Figura 1), denominados como montantes, soleiras, vergas e contravergas (SHIGUE, 2018). O preenchimento das paredes é feito com placas de OSB, e entre elas estão todas as instalações necessárias para a utilização da edificação (hidrossanitária, elétrica, de gás etc.) Edifícios construídos com o sistema wood frame têm ganhado cada vez mais espaço no setor da construção, devido ao seu rápido e fácil processo de fabricação e montagem fora do local da obra (Figura 2) (ESTRELLA et al., 2021).

\subsection{Log home}

O sistema log home (Figura 3) utiliza de madeiras roliças ou modeladas, que montadas sobrepostas umas às outras, formam uma estrutura autoportante por meio de encaixes (SHIGUE, 2018). Esse sistema dispensa o uso de pilares e vigas, e geralmente empregam a madeira em sua forma mais bruta, realizando apenas um tratamento na madeira para prevenir deterioração biológica.

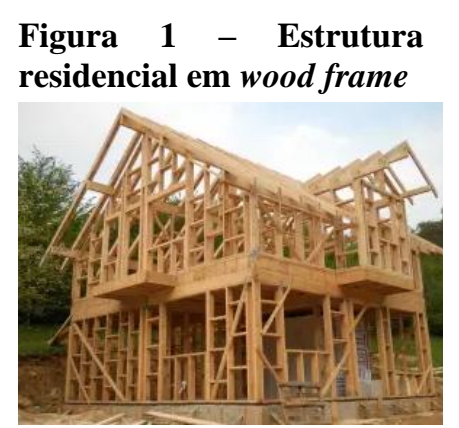

Fonte: TWBRAZIL (2019)
Figura 2 - Montagem em galpão de edificações em wood frame

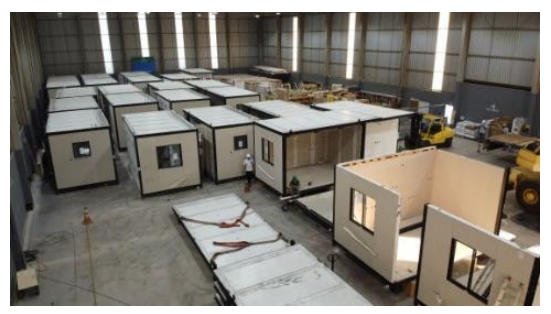

Fonte: TECVERDE ENGENHARIA (2016)
Figura 3 - Casa de campo em log home

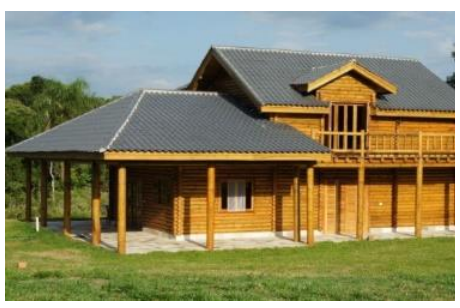

Fonte: CASABELLA (2019)

\subsection{Madeira laminada cruzada (CLT)}

A madeira laminada cruzada, em inglês, cross-laminated timber (CLT), é definida como uma madeira engenheirada sólida, composta por três a sete camadas ortogonais de madeira serrada (WANG; GONG \& CHUI, 2015). Com excelente desempenho acústico e térmico, além de uma elevada resistência ao fogo, os painéis de CLT são compostos de várias camadas individuais de laminação de madeira (Figura 4), que são secas em estufa, empilhadas em direções alternadas, coladas com adesivos estruturais e prensadas, de modo a formar um painel sólido, reto e retangular (APA, 2019; PERSSON et al., 2021). A composição em camadas orientadas transversalmente permite a fabricação de grandes 
painéis, com alta rigidez e capacidade de carga em relação ao seu peso (PERSSON et al., 2021), podendo ser utilizados em lajes e paredes (Figuras 5 e 6).

Figura 4 - Elemento de CLT

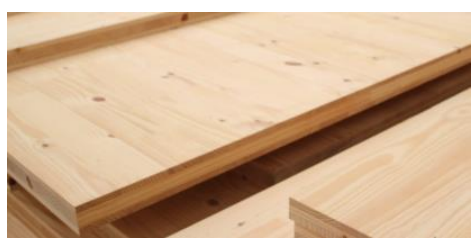

Fonte: CROSSLAM (2020)
Figura 5 - Elementos estruturais em CLT

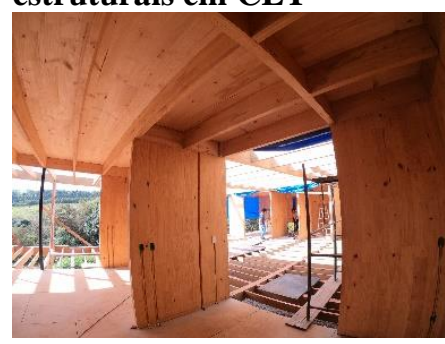

Fonte: CROSSLAM (2020)

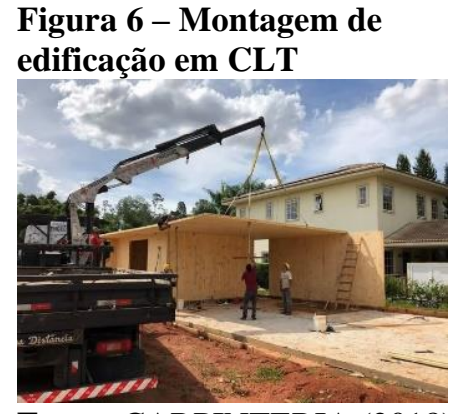

Fonte: CARPINTERIA (2018)

\subsection{Estrutura pilar-viga}

O sistema em estrutura pilar-viga consiste na concepção de edifícios onde os elementos estruturais são independentes das paredes de vedação, e a transferência de esforços se dá das vigas para os pilares. Esses produtos em madeira são produzidos a partir da extração de folheados ou flocos de madeira seca, que são unidos entre si e, subsequentemente são beneficiados em tamanhos específicos, que resultam em elementos que superam a madeira serrada convencional quando são solicitados sob esforços e no desempenho contra empenamentos e rachaduras (APA, 2019).

A vedação pode ser composta por painéis encaixados na horizontal, tábuas ou pranchas de madeira (PIZZONI, 2017). Elementos estruturais em madeira engenheirada que são encontrados no Brasil: MLC, NLT, LSL e LVL. Como painel de fechamento, o OSB é a tecnologia mais utilizada.

\subsubsection{Madeira laminada colada (MLC)}

Também conhecida como Glulam, a madeira laminada colada (glued laminated timber) representa um material mais forte que o aço e, com maior resistência e rigidez que a madeira convencional com dimensões comparáveis (APA, 2019). Pilares e vigas de MLC são produtos estruturais de madeira engenheirada (Figura 7), concebidos pela colagem das madeiras em paralelo, com adesivos duráveis e com resistência à umidade, e são amplamente utilizados em estruturas de madeira (KILINCARSLAN \& TURKER, 2021). Os grãos das laminações de madeira são colados em paralelo ao comprimento da peça final, permitindo seu uso como uma simples viga em uma estrutura residencial, ou ainda para arcos para telhados abobadados com mais de 150 metros de comprimento (Figura 8) (APA, 2019).

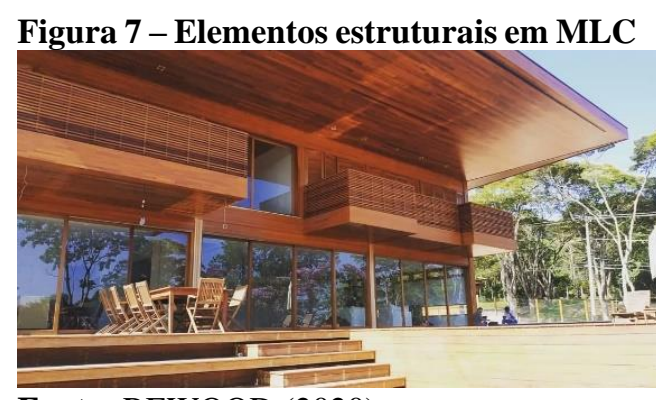

Fonte: REWOOD (2020)

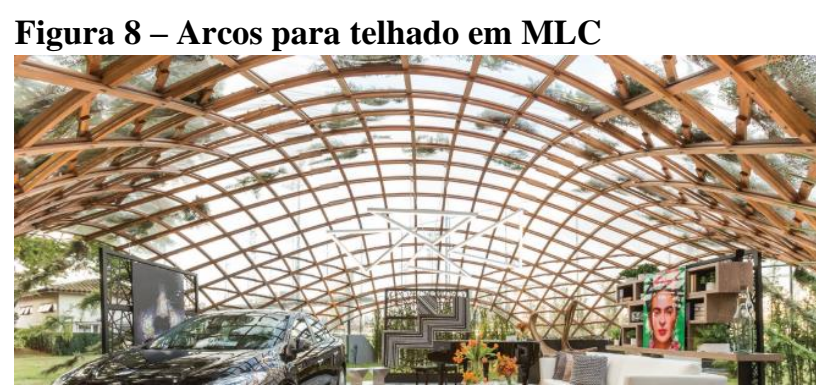

Fonte: REWOOD (2020)

\subsubsection{Madeira laminada pregada (NLT)}

A madeira laminada pregada (nail laminated timber), é produzida a partir de diversas peças de madeira serrada, que são fixadas mecanicamente com pregos ou parafusos (Figura 9), tornando a peça final um elemento estrutural único, de altíssima resistência 
(THINK WOOD, 2017). A NLT está cada vez mais sendo utilizada em edificações comerciais e residenciais, como um excelente substituto da laje de concreto e de deck em estrutura metálica (Figura 10), criando uma estética inovadora (STRUCTURECRAFT BUILDERS, 2017).

Figura 9 - Elemento estrutural em NLT

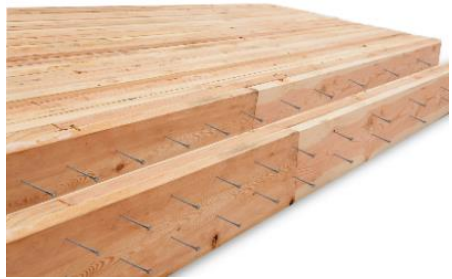

Fonte: THINK WOOD (2017)

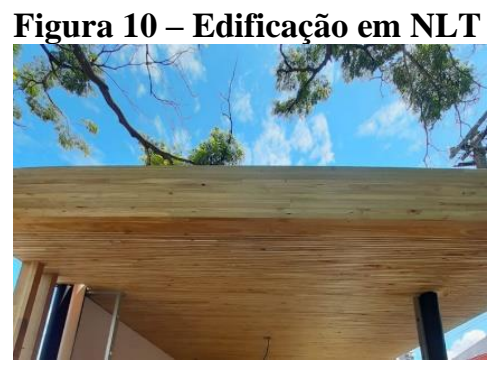

Fonte: REWOOD (2020)

\subsubsection{Madeira laminada serrada (LSL)}

A madeira laminada serrada (laminated strand lumber) é fabricada com flocos de madeira com espessura entre 0,6 a 1,3 mm e comprimento de até $300 \mathrm{~mm}$ (Figura 11) (FERRAZ et al., 2008). Combinados com o adesivo, os flocos são orientados paralelamente (APA, 2019). Sua matéria-prima não se limita a madeiras comercialmente utilizadas para elementos em madeira engenheirada, como Pinus e eucalipto, podendo serem empregadas espécies de árvores pouco utilizadas e de rápido crescimento (FERRAZ et al., 2008).

\subsubsection{Madeira microlaminada (LVL)}

A madeira microlaminada (laminated veneer lumber) compõe elementos estruturais a partir de lâminas de madeira de espessura entre 2,5 a 3,2 mm, que são criteriosamente selecionadas e sobrepostas (Figura 12), de modo que as fibras da madeira sejam coladas paralelas ao sentido longitudinal da peça (SHIGUE, 2018). Por ser fabricado com lâminas com juntas chanfradas ou lapidadas, a LVL permite fabricar elementos com comprimentos muito maiores que os da madeira serrada convencional (APA, 2019). São largamente utilizadas para a confecção de vigas.

\subsubsection{Chapa de tiras de madeira orientadas (OSB)}

Dentro da indústria de materiais de construção, a OSB (oriented strand board), surgiu como um composto à base de madeira ecologicamente correto. Sua fabricação é executada com pequenos pedaços de madeira, com diâmetro de 8 a $10 \mathrm{~cm}$ (Figura 13), que são processados, fatiados e raspados mecanicamente, para posterior secagem. Em seguida, o material é misturado, classificado quanto a sua dimensão e prensado a quente, formando uma placa estruturalmente orientada (WANG et al., 2019). Dentre suas qualidades estão: boa estabilidade dimensional, uniformidade, alta resistência à flexão e bom isolamento térmico.

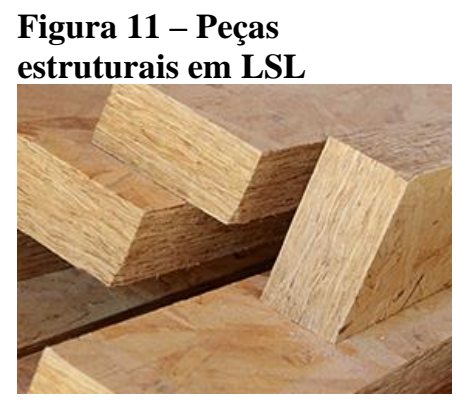

Fonte: APA (2019)

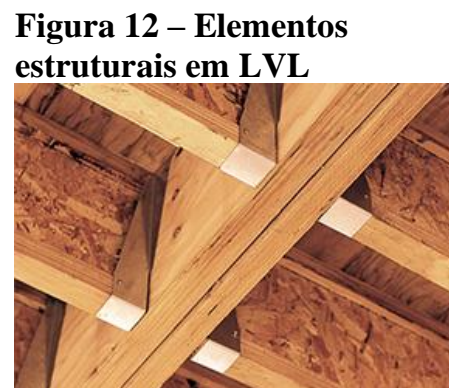

Fonte: APA (2019)
Figura 13 - Chapas de OSB

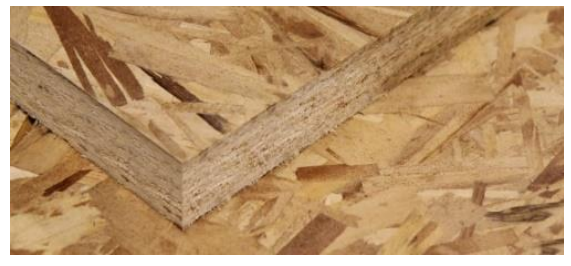

Fonte: APA (2019) 


\section{RELAÇÃO DAS EMPRESAS QUE ATUAM NO MERCADO DE SOLUÇÕES TECNOLÓGICAS EM MADEIRA INDUSTRIALIZADA NO BRASIL}

Considerando as soluções tecnológicas listadas anteriormente, na pesquisa realizada foram identificadas dez empresas que fabricam os sistemas construtivos industrializados em madeira (Quadro 1), e seis empresas que atuam na concepção e execução dos sistemas construtivos (Quadro 2).

Quadro 1 - Fabricantes de soluções tecnológicas em madeira industrializada

\begin{tabular}{|c|c|c|c|}
\hline Empresa & \begin{tabular}{|l} 
Tecnologia \\
empregada
\end{tabular} & Localização & Descrição \\
\hline AMATA & CLT e MLC & São Paulo - SP & $\begin{array}{l}\text { Empresa que atua na construção civil de madeira } \\
\text { engenheirada há mais de } 15 \text { anos, sendo pioneira da } \\
\text { tecnologia em larga escala no Brasil. É signatária da } \\
\text { Política para associações de organizações com FSC®. }\end{array}$ \\
\hline ArtPINE & MLC & São Carlos - SP & $\begin{array}{l}\text { Faz parte de um grupo empresarial fundado há } 25 \text { anos, } \\
\text { que incorpora o uso racional da madeira como matéria- } \\
\text { prima para elementos estruturais e decorativos na } \\
\text { construção civil. Desde } 2006 \text { fabrica produtos com } \\
\text { MLC. }\end{array}$ \\
\hline $\begin{array}{l}\text { Catalana } \\
\text { Artefatos de } \\
\text { Madeira } \\
\end{array}$ & MLC & Catalão - GO & $\begin{array}{l}\text { Dedicada ao corte e serragem de madeira em grande } \\
\text { capacidade de produção, oferecem em seu portfólio: } \\
\text { madeira serrada e tratada, decks e vigas de MLC. }\end{array}$ \\
\hline $\begin{array}{l}\text { CG Sistemas } \\
\text { Construtivos }\end{array}$ & CLT e MLC & Suzano - SP & $\begin{array}{l}\text { Produz componentes modulares e customizáveis, } \\
\text { fabricando vigas de escoramento (Vigas H20), vigas } \\
\text { laminadas coladas, elementos em CLT e edificações } \\
\text { temporárias para canteiros de obras, escolas, refeitórios } \\
\text { e ambulatórios. }\end{array}$ \\
\hline $\begin{array}{l}\text { Crosslam } \\
\text { Brasil }\end{array}$ & CLT e MLC & Suzano - SP & $\begin{array}{l}\text { Empresa que fabrica e comercializa produtos de madeira } \\
\text { engenheirada e sistemas construtivos. Já atuou na } \\
\text { construção de residências, estúdios, arquibancadas, } \\
\text { igrejas e galpões. }\end{array}$ \\
\hline $\begin{array}{l}\text { Ekomposit do } \\
\text { Brasil }\end{array}$ & LVL & Lages - SC & $\begin{array}{l}\text { Possui uma planta industrial com capacidade de } \\
\text { processar anualmente cerca de } 100 \text { mil metros cúbicos } \\
\text { de madeira. Dentre seus produtos estão: vigas, pilares, } \\
\text { decks, painéis e revestimentos. }\end{array}$ \\
\hline LP Brasil & LSL e OSB & São Paulo - SP & $\begin{array}{l}\text { Subsidiária da empresa global LP Corp, líder em } \\
\text { tecnologia OSB. Atua no mercado brasileiro desde } \\
\text { 2008, quando inaugurou sua planta fabril, promovendo } \\
\text { o sistema de construção seca Frame Building System. }\end{array}$ \\
\hline $\begin{array}{l}\text { Madeiras } \\
\text { Status }\end{array}$ & MLC & Igrejinha - RS & $\begin{array}{l}\text { Empresa consolidada no ramo de madeiras tratadas. É } \\
\text { proprietária de mais } 5000 \text { hectares de florestas de Pinus } \\
\text { e eucalipto. Possui equipe técnica para projetos e } \\
\text { gerenciamento de obras no sistema log home. }\end{array}$ \\
\hline $\begin{array}{l}\text { Rewood } \\
\text { Soluções } \\
\text { Estruturais } \\
\text { em Madeira } \\
\end{array}$ & MLC e NLT & $\begin{array}{l}\text { Taboão da } \\
\text { Serra - SP }\end{array}$ & $\begin{array}{l}\text { Trabalha desde o estudo da viabilidade e projeto do } \\
\text { empreendimento, até a fabricação do material, } \\
\text { usinagem, transporte e montagem dos elementos } \\
\text { estruturais. }\end{array}$ \\
\hline TWBrazil & MLC e OSB & $\begin{array}{l}\text { Ponta Grossa - } \\
\text { PR }\end{array}$ & $\begin{array}{l}\text { Fundada em 2001, sob a perspectiva de atender a } \\
\text { demanda por madeiras engenheiras de florestas } \\
\text { plantadas. Comercializa elementos construtivos a base } \\
\text { de Pinus, eucalipto e teca. }\end{array}$ \\
\hline
\end{tabular}

Fonte: Os autores (2021) 
Quadro 2 - Empresas que atuam na concepção e execução de sistemas construtivos industrializados em madeira

\begin{tabular}{|c|c|c|c|}
\hline Empresa & $\begin{array}{l}\text { Tecnologia } \\
\text { empregada }\end{array}$ & Localização & Descrição \\
\hline Carpinteria & CLT & São Paulo - SP & $\begin{array}{l}\text { Empresa especializada em engenharia estrutural em } \\
\text { madeira, possuindo escritório de projetos, laboratórios } \\
\text { para perícias e consultorias, e profissionais para } \\
\text { montagem de estruturas. Ganhadora de } 2 \text { prêmios } \\
\text { Talento. }\end{array}$ \\
\hline Casabella & Log home & São Carlos - SP & $\begin{array}{l}\text { Fundada em } 2000 \text {, a empresa trabalha com projetos } \\
\text { únicos e sob medida. Utiliza como matéria-prima Pinus } \\
\text { e eucalipto provenientes de reflorestamento. }\end{array}$ \\
\hline K.Bana & Wood frame & Curitiba - PR & $\begin{array}{l}\text { Empresa especializada em fabricação de cabanas para } \\
\text { campo e hotéis fazenda, através de modelos pré- } \\
\text { estabelecidos. É responsável por todas as fases do } \\
\text { projeto, desde o estudo topográfico, até o acabamento } \\
\text { final. }\end{array}$ \\
\hline Reallogs & Log home & $\begin{array}{l}\text { Itamarandiba - } \\
\mathrm{MG}\end{array}$ & $\begin{array}{l}\text { Há mais de } 20 \text { anos no mercado, surgiu como usina de } \\
\text { tratamento em madeira reflorestada. Hoje atua no } \\
\text { aperfeiçoamento do eucalipto tratado, permitindo a } \\
\text { construção de galpões, pontes, passarelas e telhados. }\end{array}$ \\
\hline $\begin{array}{l}\text { TECVERDE } \\
\text { Engenharia }\end{array}$ & Wood frame & Araucária - PR & $\begin{array}{l}\text { Fundada em 2009, é referência em estruturas em } \\
\text { madeira no Brasil, sendo detentora da maior planta } \\
\text { fabril em wood frame no território nacional. Possui } \\
\text { como experiência mais de } 175.000 \mathrm{~m}^{2} \text { construídos. }\end{array}$ \\
\hline $\begin{array}{l}\text { Tiny Houses } \\
\text { BR }\end{array}$ & Wood frame & Porangaba - SP & $\begin{array}{l}\text { Há mais de } 25 \text { anos no mercado, são especialistas em } \\
\text { projetos e construção de mini casas sob rodas, sendo } \\
\text { mais versáteis que trailers e motorhomes. }\end{array}$ \\
\hline $\begin{array}{l}\text { Zenóbio } \\
\text { Madeiras }\end{array}$ & Wood frame & Armazém - SC & $\begin{array}{l}\text { Produz componentes estruturais em wood frame a partir } \\
\text { de Pinus de reflorestamento, como montantes, barrotes, } \\
\text { vigas, caibros e lamelados colados. }\end{array}$ \\
\hline
\end{tabular}

Fonte: Os autores (2021)

\section{CONSIDERAÇÕES FINAIS}

O presente estudo propôs um levantamento das soluções tecnológicas dos sistemas construtivos industrializados em madeira no Brasil, através do levantamento de algumas empresas atuantes na área. Verificou-se que as tecnologias em madeira engenheirada e demais sistemas construtivos em madeira estão se consolidando no território brasileiro, com diversas empresas bem estabilizadas e com a forte proposta de expandir esse tipo de construção, especialmente pelas vantagens que um sistema off-site oferece: racionalização de material e mão-de-obra, agilidade na fase de construção e economia dos custos totais.

Entretanto, a construção industrializada em madeira engenheirada está maciçamente localizada nas regiões Sul e Sudeste do Brasil, regiões com o maior índice de industrialização, e pouco difundida nas demais regiões. Então, espera-se que o presente trabalho possa ser o ponto de partida para demais pesquisas específicas sobre o assunto, contribuindo para a divulgação da construção off-site em madeira.

\section{REFERÊNCIAS}

ABDI - AGÊNCIA BRASILEIRA DE DESENVOLVIMENTO INDUSTRIAL. Manual da construção industrializada. 2015. v. 1, p. 1-115.

APA - THE ENGINEERED WOOD ASSOCIATION. Structural composites lumber (SCL). 2019. Disponível em: < https://www.apawood.org/structural-composite-lumber>. Acesso em: 15 mar. 2021.

CARPINTERIA. Madeira laminada colada (CLT): produção e desnvolvimento. 2018. Disponível em: <https://carpinteria.com.br/2018/04/08/madeira-laminada-cruzada-clt/>. Acesso em: 15 mar. 2021. 
CASABELLA. Casas de madeira ecologicamente corretas. 2019. Disponível em: <http://casabella.etc.br/>. Acesso em: 09 abr. 2021.

CROSSLAM. CLT - Cross laminated timber. 2020. Disponível em: https://www.crosslam.com.br/site/clt/>. Acesso em: 15 abr. 2021.

ESTRELLA, X.; MALEK, S.; ALMAZÁN, J. L.; GUINDOS, P.; MARÍA, H. S. Experimental study of the effects of continuous rod hold-down anchorages on the cyclic response of wood frame shear walls. Engineering Structures, v. 230, p. 1-15, 2021.

KILINCARSLAN, S.; TURKER, Y. S. Experimental investigation of the rotational behavior of glulam column beam joints reinforced with fiber reinforced polymer composites. Composite Structures, v. 262, p. 1-8, 2021.

KUZMAN, M. K.; KLARIC, S.; BARCIC, A. P.; VLOSKY, R. P.; JANAKIESKA, M. M.; GROSELJ, P. Architect perceptions of engineered wood products: An exploratory study of selected countries in Central and Southeast Europe. Construction and Building Materials, v. 179, p. 360-370, 2018.

MORADPOUR, P.; PIRAYESH, H.; GERAMI, M.; JOUYBARI, I. R. Laminated strand lumber (LSL) reinforced by GFRP; mechanical and physical properties. Construction and Building Materials, v. 158, p. 236-242, 2018.

PERSSON, P.; FLODÉN, O.; DANIELSSON, H.; PEPLOW, A.; ANDERSEN, L. V. Improved low-frequency performance of cross-laminated timber floor panels by informed material selection. Applied Acoustics, v. 179, p. 1-14, 2021.

PIZZONI, C. P. Vedações verticais externas do sistema plataforma em madeira: medidas para manutenção. Orientador: Ângela do Valle. 2017. 169 p. Dissertação (Mestre em Arquitetura e Urbanismo) - Universidade Federal de Santa Catarina, Florianópolis, 2017.

RESCALVO, F. J.; DURIOT, R.; POT, G.; GALLEGO, A.; DENAUD, L. Enhancement of bending properties of Douglas-fir and poplar laminate veneer lumber (LVL) beams with carbon and basalt fibers reinforcement. Construction and Building Materials, v. 263, p. 1-9, 2020.

REWOOD. Cases. 2020. Disponível em: <https://rewood.com.br/cases>. Acesso em: 15 abr. 2021.

SHIGUE, E. K. Difusão da Construção em Madeira no Brasil: Agentes, Ações e Produtos. Orientador: Lucia Zanin Shimbo. 2018. 250 p. Dissertação (Mestre em Ciências) - Universidade de São Paulo, São Carlos, 2018.

STRUCTURECRAFT BUILDERS. Nail laminated timber - NLT. 2017. Disponível em: <https://structurecraft.com/materials/mass-timber/nail-laminated-timber>. Acesso em: 23 mar. 2021.

TECVERDE ENGENHARIA. Construções eficientes. 2016. Disponível em: <https://www.tecverde.com.br/>. Acesso em: 07 abr. 2021

THINK WOOD. Hail to the nail! 2017. Disponível em: <https://www.thinkwood.com/masstimber/nlt>. Acesso em: 15 mar. 2021.

TUMELERO, N. Pesquisa documental: conceito, exemplos e passo a passo, 2019. Disponível em: <https://blog.mettzer.com/pesquisa-documental/>. Acesso em: 15 abr. 2021.

TWBRAZIL. Wood frame. $2019 . \quad$ Disponível em: <https://www.twbrazil.com.br/arquivos/catalogo/wood-frame>. Acesso em: 15 abr. 2021.

WANG, Z.; GONG, M.; CHUI, Y. Mechanical properties of laminated strand lumber and hybrid cross laminated timber. Construction and Building Materials, v. 101, p. 622-627, 2015.

\section{AGRADECIMENTOS}

À Coordenação de Aperfeiçoamento de Pessoal de Nível Superior - Brasil (CAPES) pelo apoio à pesquisa e publicação. 